\title{
Culture of domestic cat ovarian tissue in vitro and in the chick embryo chorioallantoic membrane
}

\author{
J.M.V. Vilela ${ }^{\text {a }}$, E.C.R. Leonel ${ }^{\text {b }}$, L. D'Oliveira ${ }^{\text {a }}$, R.E.G. Paiva ${ }^{\text {a }}$, \\ A.L. Miranda-Vilela ${ }^{\text {c,d }}$, C.A. Amorim ${ }^{\mathrm{e}}$, A. Pic-Taylor ${ }^{\mathrm{c}}$, C.M. Lucci ${ }^{\mathrm{a}, *}$ \\ a Instituto de Ciências Biológicas, Departamento de Ciências Fisiológicas, Universidade de Brasília, Brasília, DF, Brazil \\ ${ }^{\mathrm{b}}$ Universidade Estadual Paulista - UNESP, Instituto de Biociências, Letras e Ciências Exatas, Departamento de Biologia, Laboratório \\ de Microscopia e Microanálises, São José do Rio Preto, São Paulo, Brazil \\ ${ }^{\mathrm{c}}$ Departamento de Genética e Morfologia, Universidade de Brasília, Brasília, DF, Brazil \\ ${ }^{\mathrm{d}}$ Faculdades Integradas da União Educacional do Planalto Central (Faciplac), Curso de Medicina, Campus Gama, DF, Brazil \\ e Université Catholique de Louvain, Pôle de Recherche en Gynécologie, Institut de Recherche Expérimentale et Clinique, Brussels, \\ Belgium
}

\section{A R T I C L E I N F O}

\section{Article history:}

Received 25 November 2015

Received in revised form 24 May 2016

Accepted 27 May 2016

\section{Keywords:}

Viability

Cell proliferation

Development

Feline

Culture in ovo

Preantral follicle

\begin{abstract}
A B S T R A C T
In vitro culture and transplantation procedures are essential protocols employed in the evaluation of ovarian follicle survival and development. Culture in the chorioallantoic membrane (CAM) of chick embryos is an intermediate method that provides important follicle development information and has not been tested for cat ovaries to date. The aim of this study was to investigate if in vitro and CAM culture could be used as short-term systems to study cat ovarian tissue development. The ovaries of eight cats were dissected into 3- $\mathrm{mm}^{3}$ cubes, cultured in vitro and in CAM for up to 5 days, and stained with hematoxylin-eosin and Gomori trichrome. Cell proliferation was analyzed using anti-Ki67. Possible differences among groups were investigated by analysis of variance or the Kruskal-Wallis test followed by Bonferroni correction. The T-test or Wilcoxon test was used to verify differences between the CAM and IVC. Results revealed that $87.5 \%$ of all follicles were primordial during culture. The percentage of primordial follicles in the morphologically normal follicles (MNF) pool was always higher than $80 \%$, with the exception of Day 3 of CAM culture, but the number of MNF reduced significantly from Day 0 (600 out of 777 follicles) to Day 5 in the CAM (91 out of 171) and IVC (296 out of 686). The number of primordial follicles in $1 \mathrm{~mm}^{3}$ in Days 2, 3, and 5 in the CAM was significantly lower than that in the control (Day 0 ). No cellular proliferation was observed in culture. Vascularization occurred in the CAM culture, but with no association to follicular viability. In addition, both methods showed an increase in connective tissue during culture. Although no significant differences were observed in the percentage of MNF, there was a reduction in the total number of follicles, both for IVC and CAM-cultured ovarian tissue. Furthermore, anti-Ki67 did not stain any follicle after Day 0 in IVC or in CAM culture. Neither system was capable of promoting follicle growth and/or development. The results show that the CAM is not a suitable system for feline ovarian tissue and highlight the necessity to improve IVC systems in cats.
\end{abstract}

(c) 2016 Elsevier Inc. All rights reserved.

\footnotetext{
* Corresponding author. Tel.: +55 61 3107-3113; fax: +55 61 31072904.

E-mail address: carollucci@gmail.com (C.M. Lucci).
}

\section{Introduction}

Assisted reproductive technology is considered a significant tool for the conservation of threatened animal species [1,2]. With the exception of domestic cats, all other 
felids are present on the International Union for the Conservation of Nature (IUCN) Red list of endangered species [3], primarily because of human impacts such as hunting, habitat destruction, and diseases [4]. Owing to phylogenetic proximity between species, the domestic cat is an excellent model for feline reproduction studies, contributing to the understanding of felid reproductive physiology despite considerable species differences [5]. Ovarian tissue utilization has been studied for several decades with the expectation of improving preantral follicle use.

Despite significant efforts, IVC systems are not yet fully developed, at least not for most animal species. There are various difficulties in the development of an IVC system that foster preantral follicle development, such as a naturally long growth period required for such structures to reach the antral follicle stage, a period involving several growth factors and hormones [6-8]. Ovarian tissue transplantation is probably the most effective tool to evaluate follicle survival and development as it is the form closest to the physiological condition. However, grafting involves the use of immunosuppressed or immunodeficient animals (xenografts), or the availability of the same animal from which the tissue was removed (autograft) [9,10]. In contrast, IVC is easily performed in the laboratory without host animals although it does not mimic the physiological environment of the tissue. Indeed, cells can only rely on the nutrients and growth factors available in the culture medium, with all necessary exchanges occurring by diffusion as there are no blood vessels [11].

Culture in the chorioallantoic membrane (CAM) of chick embryos can be considered as an intermediate method for tissue evaluation as it allows the re-establishment of blood supply and affords the grafted tissue access to growth factors available for chick embryo development. Despite being a less controlled system than IVC, it is not as invasive as transplantation and involves simpler implementation. Moreover, the lack of a functional immune system before Day 17 of chick embryo development prevents graft rejection [12].

Although there have been satisfactory results of ovarian tissue IVC in some species [11,13-18], only a few studies in cats have been reported [19-24] and the system is not yet fully developed. Culture in the CAM has been performed in mice [25], bovine [25,26], and human [11,13,27,28] ovarian tissue. In cats, it has been used as model for studying the growth of the feline vaccine-associated fibrosarcoma cell line [29], but it has not been tested for ovarian tissue development to date. Therefore, the aim of this study was to investigate if in vitro and CAM culture could be used as short-term systems to study cat ovarian tissue development.

\section{Methods}

\subsection{Ovary collection}

The ovaries of eight healthy 1 - to 5-year-old adult queens in interestrus were collected at a veterinary clinic following elective ovariohysterectomies and transported to the laboratory in physiological solution ( $0.9 \%$ saline) at $36{ }^{\circ} \mathrm{C}$ within 1 hour. Adipose tissue and ligaments were removed. The ovaries were washed with a $70 \%$ ethanol and saline solution. Each pair of ovaries was dissected into $3 \mathrm{~mm}^{3}$ cubes with 21 fragments used.

\subsection{Experimental design}

If not specified, the reagents were obtained from SigmaAldrich.

This study was conducted in strict accordance with the applicable national and international guidelines. The protocol was approved by the Animal Experiments Ethics Committee of the University of Brasilia (permit number 30115/2013).

The ovarian pieces of each animal were distributed into the following treatment groups:

(1) Control: One piece was immediately fixed in $4 \%$ paraformaldehyde for histologic and immunohistochemical analyses.

(2) In vitro culture (IVC): Ten pieces were in vitro cultured in an incubator (Te-399, Tecnal, Brazil) at $37.5{ }^{\circ} \mathrm{C}$ and $5 \%$ $\mathrm{CO}_{2}$ (in air) in a 96-well plate. Each well contained $350 \mu \mathrm{L}$ of M-199 medium supplemented with $0.23-\mathrm{mM}$ sodium pyruvate, $2 \mathrm{mM}$ of L-Glutamine, 10\% fetal calf serum (Gibco, the Netherlands), insulin-transferrinselenium $(6.25 \mu \mathrm{g} / \mathrm{mL}, 6.25 \mu \mathrm{g} / \mathrm{mL}$, and $6.25 \mathrm{ng} / \mathrm{mL})$, 100-U/mL penicillin, 0.1-mg/mL streptomycin, and 2.3$\mu \mathrm{g} / \mathrm{mL}$ FSH (Folltropin V, Vetrepharm, Canada). Every other day, $175 \mu \mathrm{L}$ of the medium was replaced with fresh medium.

(3) CAM-culture: Ten pieces were cultured in the CAM of chick embryos as described by Martinez-Madrid et al. [27]. In summary, fertilized eggs were incubated for 3 days at $37{ }^{\circ} \mathrm{C}$ in $60 \%$ relative air humidity and rotated hourly. On Day 3 of incubation, a rectangular window $(1 \times 1.5 \mathrm{~cm})$ was made in the eggshell. Two milliliters of albumen were withdrawn using a 21-gauge needle through the large blunt end of the egg. The window was covered with a piece of tape to prevent dehydration, and the eggs replaced in the incubator without rotation. On Day 10 of incubation, a small area of the CAM was gently traumatized by laying a $1 \mathrm{~cm}^{2}$ strip of sterile silk paper on the surface of the epithelium and removing it immediately. The ovarian tissue graft was subsequently placed onto the traumatized CAM with sterile forceps and the eggshell re-covered.

For both culture systems, ovarian grafts were cultured for up to 5 days, with two pieces removed each day and fixed in $4 \%$ paraformaldehyde for histologic and immunohistochemical analyses.

\subsection{Histologic processing}

All fixed grafts were dehydrated in ethanol, clarified in xylene, and embedded in Paraplast Plus, $4 \mu \mathrm{m}$-thick semiserial cuttings were performed. Every fourth section was 
fixed onto slides and stained with hematoxylin-eosin or Gomori trichrome.

To calculate the number of follicles in $1 \mathrm{~mm}^{3}$ of ovarian tissue, we used the correction factor described by Gougeon and Chainy [30]

$N 1=\frac{N o \times S t \times T s}{S o \times d n}$

Where $N 1=$ the total number of follicles calculated per class; $N o=$ the number of follicles observed in the ovary; $S t=$ the total number of sections in the ovary; $T s=$ the thickness of the section $(\mu \mathrm{m})$; So $=$ the total number of sections observed; and $d n=$ the mean diameter of oocyte nucleus for each follicle class [31].

Primordial follicles (oocytes surrounded by a single layer of flattened granulosa cells) and growing follicles (oocytes surrounded by one or more complete layers of cuboidal granulosa cells) were classified into morphologically normal follicles (MNF) or degenerate follicles according to their morphologic appearance. Follicles were considered normal when spherical and intact, with a uniform distribution of granulosa cells and a spherical oocyte. Only follicles presenting a visible oocyte nucleus were counted.

\subsection{Immunohistochemistry}

The Ki67 proliferation marker was used to identify cell proliferation because it is only present in active phases of the cell cycle (G1, S, G2, and mitosis). Sections were deparaffinized with xylol and rehydrated in decreasing concentrations of alcohol. After blocking endogenous peroxidase activity with $3 \% \mathrm{H}_{2} \mathrm{O}_{2}$ diluted in methanol, sections were incubated for 30 minutes with $5 \%$ fat-free powdered milk diluted in PBS to block nonspecific binding sites before incubation with monoclonal mouse antihuman Ki67 antibodies (dilution 1:25, clone MIB1, ref M7240; Dako) overnight at $4{ }^{\circ} \mathrm{C}$. Slides were incubated for 60 minutes at room temperature with goat anti-mouse antibodies (dilution 1:200 in BSA 1M, IgG-HRP-HRO conjugated, ref sc-2005; Santa Cruz Biotechnology, USA) followed by avidin for 45 minutes at $37{ }^{\circ} \mathrm{C}$ (ImmunoCruz, Rabbit ABC Staining System Kit, ref. sc-2018; Santa Cruz Biotechnology, USA). Diaminobenzidine was used as a chromogen (K3468, Dako). Slides were subsequently counterstained with Mayer hematoxylin and mounted with Canada Balsam (ref 1003; Dinâmica, Brazil). Negative controls consisted of the dilution solution only. Mouse ovary was used as a positive control. Follicles with one or more Ki67-marked granulosa cell(s) were considered proliferative.

Actin- $\alpha$ antibody was used to identify vascularization in the ovaries. Sections were deparaffinized with xylol and rehydrated in decreasing alcohol concentrations. Antigen retrieval was subsequently performed in citrate buffer $10 \mathrm{mM}, \mathrm{pH} 6.0$, for 20 minutes at $93{ }^{\circ} \mathrm{C}$. Endogenous peroxidase was blocked using $10 \% \mathrm{H}_{2} \mathrm{O}_{2}$ in methanol. Nonspecific protein reactions were blocked using 5\% skimmed milk for 30 to 60 minutes. Samples were incubated with mouse monoclonal actin- $\alpha$ antibody (dilution
1:100, sc-32251, Santa Cruz Biotechnology, CA, USA) overnight at $4{ }^{\circ} \mathrm{C}$. The slides were subsequently incubated with EnVision TM + Dual Link System-HRP polymer (Dako, Carpinteria, CA, USA) for 45 minutes with diaminobenzidine used as a chromogen (K3468, Dako). Slides were then counterstained with Mayer hematoxylin and mounted with Canada Balsam (ref 1003, Dinâmica, Brazil). Negative controls were obtained by omitting the incubation with primary antibody step. Mouse prostate was used as a positive control.

\subsection{Statistical analysis}

Statistical analysis was performed using SPSS version 17.0. Quantitative variables were tested for normal distribution using the Shapiro-Wilk test and transformed to $\arcsin \sqrt{ } \%$ when not normally distributed. Possible differences among groups were investigated by analysis of variance or the Kruskal-Wallis test (data not normally distributed, even after transformation), followed by Bonferroni (multiple comparisons) or Mann-Whitney $U$ test (2-to-2 comparisons). The T-test or Wilcoxon test (data not normally distributed) was used to verify differences between the CAM and IVC (paired comparisons). Differences were considered significant when $\mathrm{P}<0.05$.

\section{Results}

The survival rate of chick embryos was 75.58\% (130 of 172) after grafting onto traumatized CAM. Chick embryo death was frst detected by the matte appearance of the CAM and yolk sac. All the analyzed ovarian fragments were from viable eggs.

A total of 4599 ovarian follicles were analyzed: 4025 primordial and 574 growing follicles (primary and secondary follicles). It was observed that primordial follicles were always in the majority (87.5\%) of all follicles found. There was a general decrease in the percentage of both primordial and growing MNF during culture (Table 1). However, significant differences in the percentage of MNF were only observed for IVC compared to the control (Day 0)

\section{Table 1}

Mean percentage \pm standard deviation and total number of morphologically normal (MN) primordial and growing follicles in the control (Day 0) and during 5 days of culture in vitro (IVC) and in the CAM.

\begin{tabular}{lllll}
\hline Treatment & Day & $\begin{array}{l}\text { \% MN primordial } \\
\text { follicles }\end{array}$ & $\begin{array}{l}\text { \% MN growing } \\
\text { follicles }\end{array}$ & $\begin{array}{l}\text { Total } \\
\text { follicles }\end{array}$ \\
\hline Control & 0 & $79 \pm 8(600 / 777)$ & $89 \pm 16(35 / 44)$ & 821 \\
& 1 & $60 \pm 24(351 / 637)$ & $69 \pm 32(31 / 43)$ & 680 \\
& 2 & $38 \pm 20(127 / 285)$ & $59 \pm 39(19 / 43)$ & 328 \\
CAM & 3 & $60 \pm 26(110 / 191)$ & $65 \pm 5(55 / 86)$ & 277 \\
& 4 & $60 \pm 30(165 / 340)$ & $57 \pm 37(29 / 43)$ & 383 \\
& 5 & $53 \pm 41(91 / 171)$ & $44 \pm 62(15 / 18)$ & 189 \\
& 1 & $56 \pm 20(501 / 864)$ & $54 \pm 39(39 / 78)$ & 942 \\
IVC & 2 & $53 \pm 12^{\mathrm{a}}(314 / 581)$ & $43 \pm 35^{\mathrm{a}}(28 / 51)$ & 632 \\
& 3 & $49 \pm 18^{\mathrm{a}}(379 / 745)$ & $47 \pm 34^{\mathrm{a}}(58 / 119)$ & 864 \\
& 4 & $46 \pm 17^{\mathrm{a}}(222 / 443)$ & $14 \pm 22(4 / 51)$ & 468 \\
& 5 & $42 \pm 18^{\mathrm{a}}(296 / 686)$ & $46 \pm 45(11 / 24)$ & 710 \\
\hline
\end{tabular}

Abbreviation: CAM, chorioallantoic membrane.

a Indicates significant difference from the control (Day 0) on the percentage of MN follicles $(\mathrm{P}<0.05)$. 
from Day 2 onward for primordial follicles, and on Days 2 and 3 for growing follicles. The percentages of morphologically normal growing follicles on Days 4 and 5 of IVC did not differ from Day 0 ( $\mathrm{P}>0.05$ ), probably due to the low number of follicles and a high variation among ovarian grafts. No difference was observed between the percentages of MNF (primordial or growing) in ovarian fragments cultured in the CAM and the control.

Primordial follicles were also present in greater numbers for MNF (Table 2). There was no significant difference between the CAM and IVC over the days of culture $(\mathrm{P}>0.05)$. The percentage of primordial follicles in the MNF population was always greater than $80 \%$, except for Day 3 of cultures in the CAM, where there was a large variation among samples and many grafts did not present any follicle. Moreover, on Day 3 of both IVC and the CAM, the percentage of growing follicles was higher than that in the control although no statistical difference was observed ( $\mathrm{P}>0.05)$.

The estimated number of follicles in $1 \mathrm{~mm}^{3}$ of ovarian tissue is presented in Table 3. The number of primordial follicles in $1 \mathrm{~mm}^{3}$ in Days 2, 3, and 5 in the CAM was significantly lower $(\mathrm{P}<0.05)$ than that in the control (Day $0)$. No other statistical difference was observed.

The most frequently observed alterations in primordial follicles were oocytes with a pyknotic nucleus, retracted oocyte cytoplasm, and follicle detached from the surrounding stroma. Other degeneration signs were oocytes detached from granulosa cells, cytoplasmic vacuoles in the oocyte, and granulosa cell disorganization/pyknosis (Fig. 1).

Figure 2 shows representative images of uncultured (control) and cultured ovarian grafts in vitro and in the CAM stained with hematoxylin-eosin (Fig. 2A-C), Ki67 marker (Fig. 2D-F), and Gomori trichrome (Fig. 2G-I). Although MNF was abundant on Day 0 (control-Fig. 2A), only few follicles were found in the CAM (Fig. 2B), with numerous degenerated follicles observed in IVC (Fig. 2C). Moreover, an increasing vascularization of tissue cultured for 5 days in the CAM (Fig. 2B) was observed, which was obviously not observed in IVC (Fig. 2C). On Day 0 (control-Fig. 2D), it was possible to observe proliferating granulosa cells in growing follicles, marked by Ki67 antibody. During culture, however, no marked cells were observed whatsoever, neither in IVC nor in CAM-cultured grafts (Fig. 2E and F). Gomori

Table 2

Mean percentage \pm standard deviation and total number of primordial and growing follicles considering only morphologically normal follicles (MNF) in Control (Day 0) and during 5 days of culture in vitro (IVC) and in the chorioallantoic membrane (CAM).

\begin{tabular}{llcr}
\hline Treatment & Day & \% Primordial/MNF & \% Growing/MNF \\
\hline Control & 0 & $94 \pm 4(600 / 635)$ & $6 \pm 4(35 / 635)$ \\
& 1 & $92 \pm 8(351 / 382)$ & $8 \pm 8(31 / 382)$ \\
CAM & 2 & $88 \pm 10(127 / 146)$ & $12 \pm 10(19 / 146)$ \\
& 3 & $66 \pm 29(110 / 165)$ & $34 \pm 29(55 / 165)$ \\
& 4 & $81 \pm 20(165 / 194)$ & $19 \pm 20(29 / 194)$ \\
& 5 & $91 \pm 16(91 / 106)$ & $9 \pm 16(15 / 106)$ \\
IVC & 1 & $94 \pm 7(501 / 540)$ & $6 \pm 7(39 / 540)$ \\
& 2 & $90 \pm 14(314 / 342)$ & $10 \pm 14(28 / 342)$ \\
& 3 & $86 \pm 23(379 / 437)$ & $14 \pm 23(58 / 437)$ \\
& 4 & $95 \pm 10(222 / 226)$ & $5 \pm 10(4 / 226)$ \\
& 5 & $97 \pm 5(296 / 307)$ & $3 \pm 5(11 / 307)$ \\
\hline
\end{tabular}

Table 3

Number (mean \pm standard deviation) of primordial and growing follicles in $1 \mathrm{~mm}^{3}$ of ovarian tissue.

\begin{tabular}{llcr}
\hline Treatment & Day & Total primordial & Total growing \\
\hline Control & 0 & $377 \pm 305$ & $15 \pm 16$ \\
& 1 & $299 \pm 288$ & $15 \pm 20$ \\
CAM & 2 & $101 \pm 132^{\mathrm{a}}$ & $13 \pm 23$ \\
& 3 & $86 \pm 149^{\mathrm{a}}$ & $24 \pm 41$ \\
& 4 & $156 \pm 334$ & $11 \pm 16$ \\
& 5 & $68 \pm 85^{\mathrm{a}}$ & $4 \pm 11$ \\
IVC & 1 & $379 \pm 331$ & $30 \pm 45$ \\
& 2 & $285 \pm 182$ & $21 \pm 22$ \\
& 3 & $360 \pm 145$ & $35 \pm 42$ \\
& 4 & $157 \pm 157$ & $8 \pm 15$ \\
\hline
\end{tabular}

${ }^{a}$ Indicates significant difference from the control (Day 0 ) on the number of follicles $(\mathrm{P}<0.05)$.

trichrome staining showed that grafts presented more and more connective tissue fibers as they remained longer in culture, irrespective of the system (Fig. 2G-I).

Vessels were observed in the CAM from Day 1 of culture, but the CAM only adhered to the ovarian tissue on Day 2 of culture. Chicken blood vessels permeating cat ovarian tissue cultured in CAM were visualized on Days 4 and 5 , as confirmed by actin- $\alpha$ antibody, containing nucleated red blood cells (Fig. 3).

\section{Discussion}

This represents the first study of the comparison of cat ovarian tissue in IVC and in the CAM. Culture in the CAM has been performed in mice, bovine [25,26], and humans $[11,27,28]$, reporting that primordial follicles survive for up to 5 days in ovo. In the present study, primordial follicles represented $80 \%$ to $95 \%$ of all the morphologically normal follicles on each day of culture. However, there was a significant reduction in the estimated number of follicles counted on Days 2, 3, and 5 of the CAM culture compared to Day 0 (control), showing that although the proportion of normal primordial follicles remained constant numerous follicles did not survive culture in ovo. This was also observed by Gigli et al. [25], who reported a significant decrease in the number of follicles (primordial, primary, and secondary) both in ovarian tissue after 2 days cultured in ovo and when cultured 2 days in ovo followed by 8 days in vitro. Conversely, Cushman et al. [26] reported that the number of follicles did not change during culture of bovine ovarian tissue in the CAM. Martinez-Madrid et al. [27] reported that human ovarian tissue presented an increase in the percentage of intermediate and primary follicles after 5 days of culture in the CAM. However, their study did not report the total number of follicles counted.

In the present study, individual variation in each graft response was noted, especially on Days 3 and 5 of culture in the CAM when the total number of follicles was dramatically reduced in comparison to the control (Day 0) (Table 1). Furthermore, many grafts did not present any follicles. Day 3 of CAM culture also exhibited an increase in the number of morphologically normal (MN) growing follicles together with a decrease in the percentage of MN primordial follicles, suggesting follicle activation and development. 

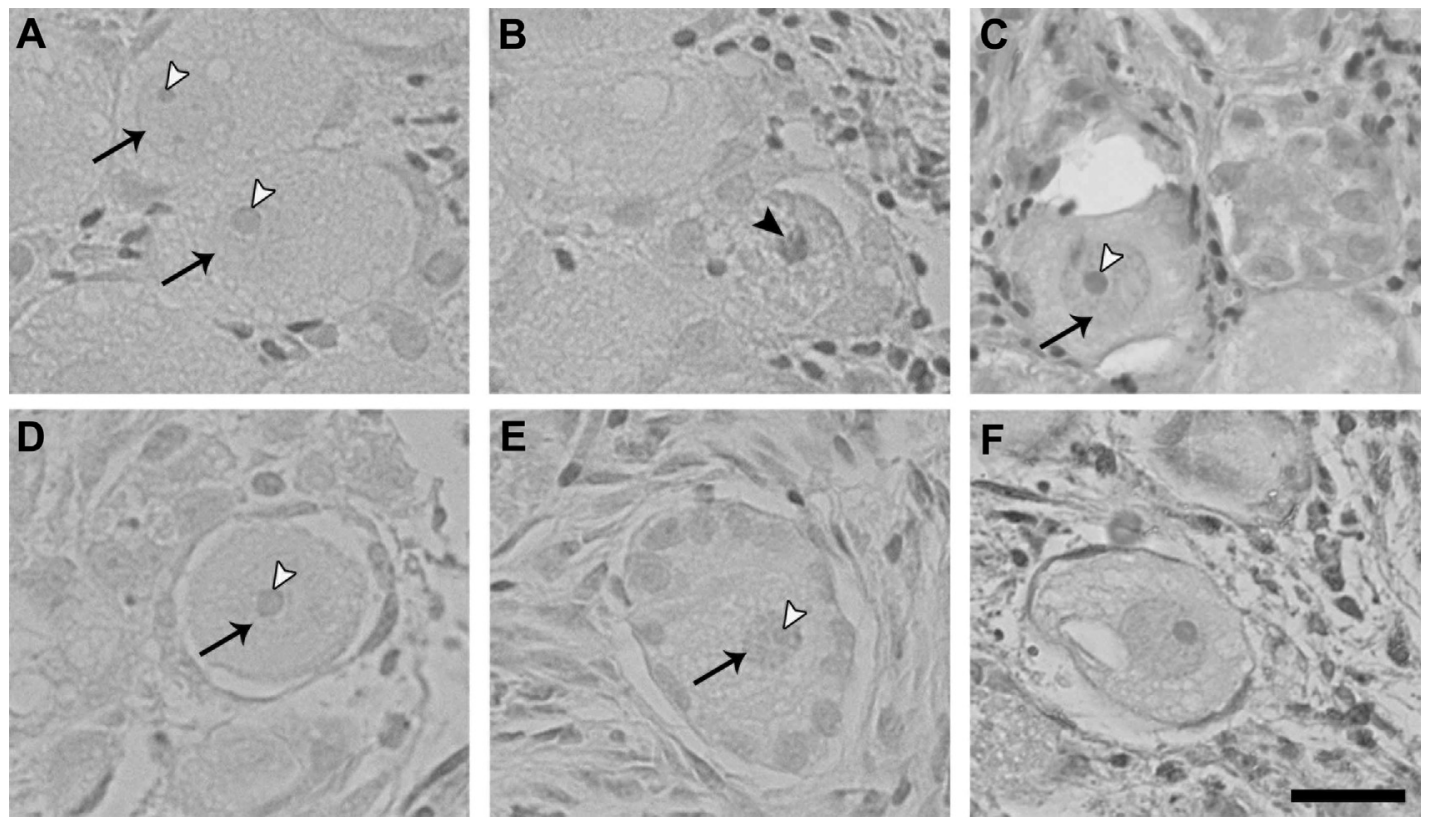

Fig. 1. More frequently observed alterations in ovarian follicles in IVC and CAM culture. Dark arrows indicate the oocyte nucleus; white arrowheads indicate nucleolus. (A) Morphologically normal primordial follicles. (B) Primordial follicle with oocyte pyknotic nucleus (dark arrowhead). (C) Primordial follicle with retracted ooplasm. (D) Primordial follicle with ooplasm detached from granulosa cells. (E) Growing follicle detached from the surrounding stroma. (F) Primordial follicle presenting cytoplasm vacuoles in the oocyte and detached from surrounding stroma. Bar $=10 \mu \mathrm{m}$. CAM, chorioallantoic membrane.

However, statistical analyses did not show any significant differences, and these results were not maintained on Days 4 and 5 of culture in the CAM.

We expected that culturing in the CAM would be more effective because of the possibility of physiological blood vessel growth and the possible presence of natural nutrient factors. In fact, we observed new vascularization within the ovarian tissue on Days 4 and 5 of culture in the CAM, confirmed by immunohistochemistry and the presence of avian (nucleated) red blood cells. Vascularization was probably induced by the initial wound-healing process caused by traumatizing the CAM before grafting [27]. This is in accordance with the literature for human ovarian tissue cultured in the CAM for the same duration [11,27]. However, the reduced number of follicles, absence of granulosa cell proliferation, and increased connective tissue fibers observed in our study suggest that this system was not efficient to maintain or promote the development of cat ovarian follicles.

There are several studies on angiogenesis factors present in the CAM (reviewed by Ribatti et al. [32]), some of which are important for follicle development, such as basic fibroblast growth factor (bFGF) and transforming growth factor $\beta$. Although the physiological relevance of bFGF to ovarian function remains under investigation several lines of evidence suggest that bFGF may play a central role in supporting growth and development of the granulosaluteal cell [33]. Many growth factors from the transforming growth factor $\beta$ superfamily are known to play important roles in ovarian folliculogenesis. However, little is known about their role in cat ovaries. Transforming growth factor $\beta$ together with activin and several bone morphogenetic proteins are involved in paracrine actions on theca cells to attenuate LH-dependent androgen production in small- to medium-sized antral follicles [34]. In cats, this growth factor may act on intermediate and antral follicle populations, by activating the Smad signaling pathway [35]. Another growth factor present in the CAM is insulin-like growth factor I (IGF-I) [36], which is known to increase the sensitivity of granulosa cells to gonadotrophins in the mammalian ovary [37], but there are no specific studies on this growth factor for cats. It is unknown if these growth factors present in the CAM share the same amino acid composition as those needed for cat follicular growth and development. Species differences may also impact the results found in the present study. Gigli et al. [25] suggested that the anti-Müllerian hormone present in the chick embryonic gonads inhibits follicle activation in mice and bovine ovarian tissue. It is possible that the same factor is affecting the development of cat follicles in the CAM culture, but further studies are necessary to understand which other factors may influence the development of cat ovarian tissue in the CAM.

The percentage of normal follicles in IVC reduced in relation to the control. On Day 3 of IVC, as well as in CAM culture, there was an increase in the percentage of growing follicles, with a concomitant decrease in primordial follicles, although it was not statistically significant. Numerous other studies reported follicular growth in vitro in other species. However, most of them tested different hormones/ growth factors, such as growth hormone [24,38], insulinlike growth factor I, epidermal growth factor, bFGF [20,22,39-41], and activin-A [24,38,42]. In cats, after a long period without publications on this line of research, new studies are emerging, but there is no consensus about the best method for culturing cat preantral follicles. A recent 

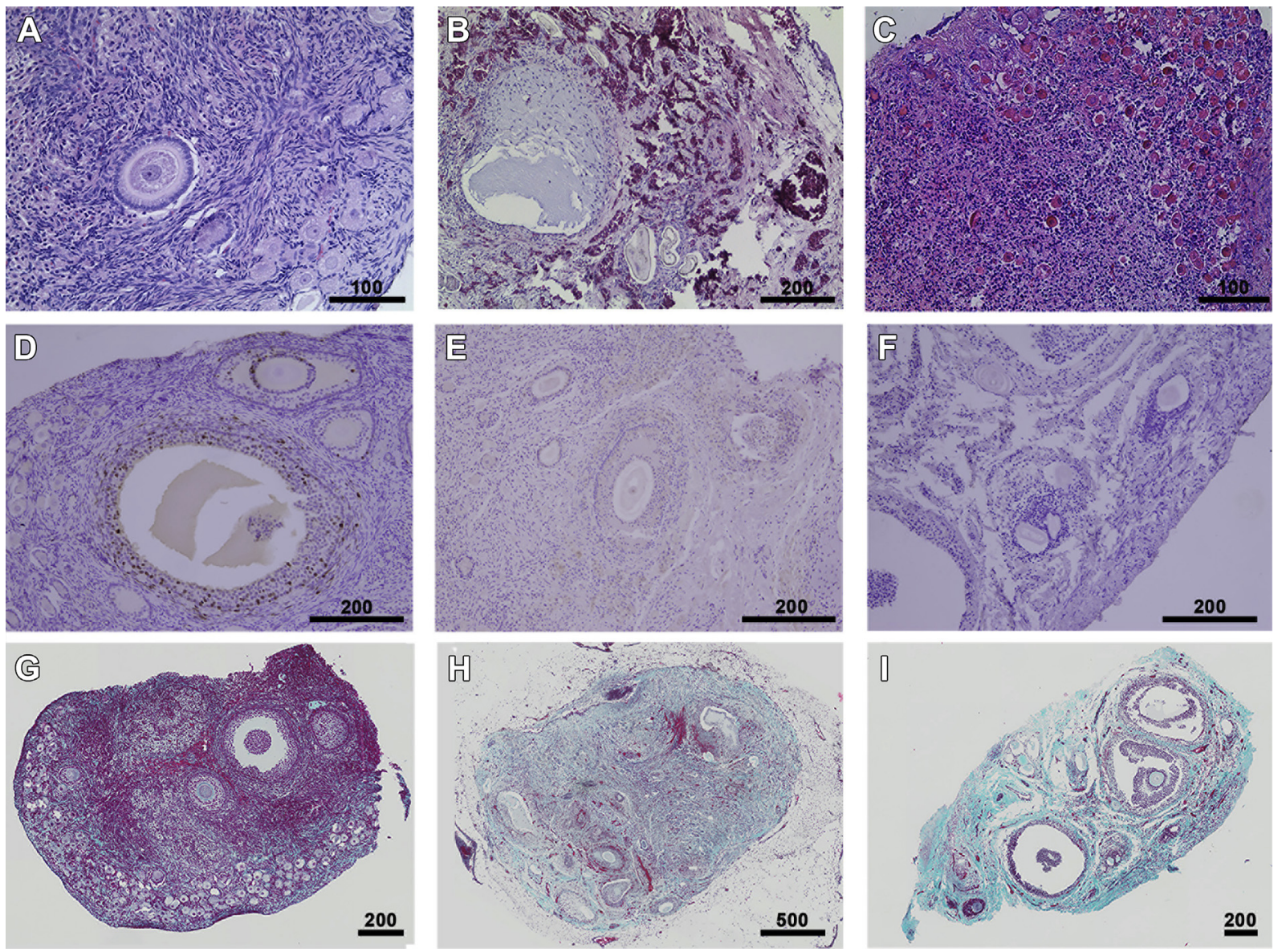

Fig. 2. Vascularization, Ki67, and Gomori trichrome in IVC and CAM culture of cat ovarian tissue. (A), (B), and (C): Vascularization of ovarian tissue on Day 0 (control-A) and Day 5 in CAM-culture (B) and IVC (C). Ovarian follicles were rarely observed after 5 days of culture in CAM; (D), (E), and (F): Cellular proliferation marked with Ki67 antibodies on Day 0 (control-D) and Day 4 of CAM-culture (E) and IVC (F). Proliferating granulosa cells stained in brown; (G), (H), and (I): Gomori trichrome staining. Control (G-Day 0) and Day 4 of CAM culture (H) and IVC (I). Cell nuclei stained in purple; blood vessels stained in red, and connective tissue fibers stained in blue. Bars $=$ scale in $\mu \mathrm{m}$. CAM, chorioallantoic membrane. (For interpretation of the references to color in this figure legend, the reader is referred to the Web version of this article.)

study reported that $85 \%$ of preantral follicles included in the ovarian tissue of domestic cats, Northern Chinese leopards and African lions were shrunken after 14 days of IVC [23]. The aforementioned study also reported a significant reduction in the number of counted follicles during culture. The culture medium used by the authors was the same as described by Telfer et al. [42], who reported that more than $90 \%$ of the follicles presented signs
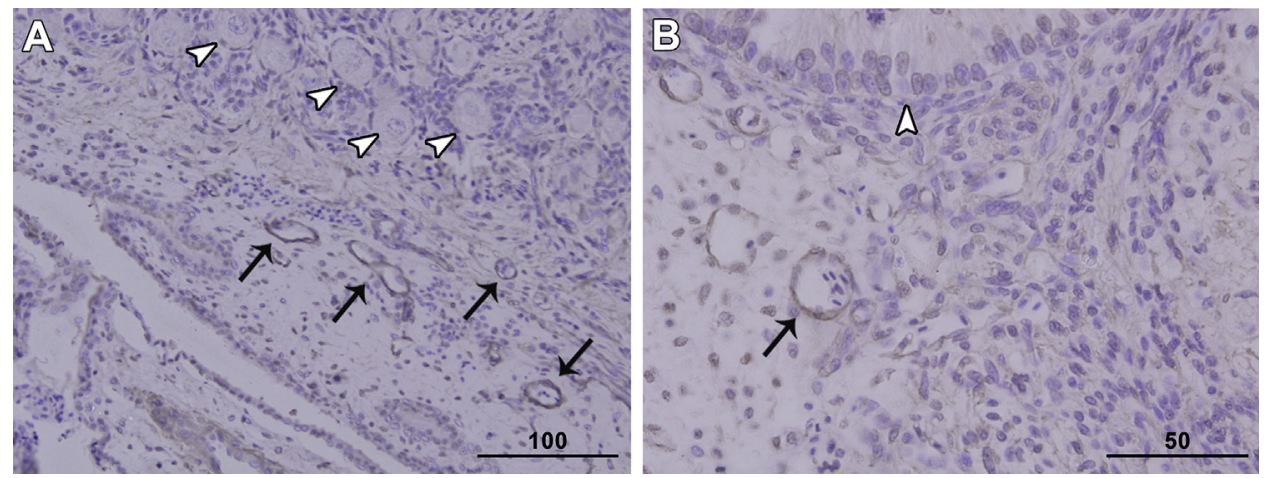

Fig. 3. Vessels marked by actin- $\alpha$ permeating the ovarian tissue. (A) Chicken blood vessels (black arrows) permeating primordial follicles (white arrowheads) in cat ovarian tissue on Day 4 of culture in the CAM. (B) Nucleated blood cells inside the vessel (black arrow) next to a secondary follicle (white arrowhead) on Day 4 of culture in the CAM. CAM, chorioallantoic membrane. 
of oocyte degeneration in human ovarian tissue cultured without activin for 4 days. Thus, it is possible that the media used in our experiments and in the literature [23] were not adequate to support the development of cat follicles. Because information on early folliculogenesis in cats is scarce $[35,43]$, it is difficult to develop an adequate culture medium.

Although results of the CAM culture and IVC did not differ statistically, the CAM culture showed a much lower number of follicles than IVC in the present study. Moreover, the variation and the number of grafts that did not present any follicles were also higher in CAM culture. These results suggest that IVC is a better option to assess follicle survival and early development than CAM. When Isachenko et al. [28] compared CAM-culture and autografting of human ovarian tissue after freezing, they observed that $88 \%$ of the primordial follicles degenerated after 5 days of CAM culture, whereas autografted ovarian tissue re-established spontaneous menstrual cycle and raised serum 17- $\beta$ estradiol from 19 to $330 \mathrm{pg} / \mathrm{mL}$ within 5 months. Autografting and xenografting of ovarian tissue have also shown promising results in other studies [44-46].

In conclusion, neither of the systems was capable of promoting follicle growth and development. The results of this study show that the CAM is not a suitable system for culturing feline ovarian tissue and highlight the necessity to improve IVC systems in cats.

\section{Acknowledgments}

The authors thank Asa Alimentos S.A. for the donation of fertilized eggs and DMV Renata Melo and Silvia Luanna from Veterinary Clinic Empório dos Bichos for the donation of cat ovaries. The authors also thank Michael Derek Taylor for conducting a grammatical review of the article. The authors wish to express their thanks to the Coordenação de Aperfeiçoamento de Pessoal de Nível Superior (CAPES) and the Conselho Nacional de Desenvolvimento Científico e Tecnológico (CNPq) for funding.

\section{References}

[1] Jewgenow K, Blottner S, Lengwinat T, Meyer HH. New methods for gamete rescue from gonads of nondomestic felids. J Reprod Fertil Suppl 1997;51:33-9.

[2] Lermen D, BlÖMeke B, Browne R, Clarke ANN, Dyce PW, Fixemer T, et al. Cryobanking of viable biomaterials: implementation of new strategies for conservation purposes. Mol Ecol 2009;18:1030-3.

[3] IUCN. The Red List of IUCN. 2014

[4] CITES. Convention on International Trade in Endangered Species of Wild Fauna and Flora. 2014.

[5] Swanson WF. Research in nondomestic species: experiences in reproductive physiology research for conservation of endangered felids. ILAR J 2003;44:307-16.

[6] Andrade ER, Silva LDM, Moraes MO, Seneda MM, Toniolli R. Cultivo in vivo de folículos pré-antrais - revisão de literatura. Revista Brasileira de Reprodução Animal 2004;28:67-72.

[7] Telfer EE. In vitro models for oocyte development. Theriogenology 1998;49:451-60.

[8] Telfer EE,Zelinski MB. Ovarian follicle culture: advances and challenges for human and nonhuman primates. Fertil Steril 2013;99:1523-33.

[9] Macedo MFD, Bezerra MB, Vicente WRR. Transplante ovariano: aplicações na reprodução de animais domésticos, silvestres e humanos. Acta Veterinaria Brasilica 2011;5:1-7.
[10] Demeestere I, Simon P, Emiliani S, Delbaere A, Englert Y. Orthotopic and heterotopic ovarian tissue transplantation. Hum Reprod Update 2009;15:649-65.

[11] Isachenko V, Mallmann P, Petrunkina AM, Rahimi G, Nawroth F, Hancke K, et al. Comparison of in vitro- and chorioallantoic membrane (CAM)-culture systems for cryopreserved medulla-contained human ovarian tissue. PLoS One 2012;7:e32549.

[12] Ribatti D. The Chick Embryo Chorioallantoic Membrane in the Study of Angiogenesis and Metastasis: The CAM assay in the study of angiogenesis and metastasis. New York, NY: Springer; 2010.

[13] Isachenko V, Montag M, Isachenko E, van der Ven K, Dorn C, Roesing B, et al. Effective method for in-vitro culture of cryopreserved human ovarian tissue. Reprod Biomed Online 2006;13:228-34.

[14] Carvalho AA, Faustino LR, Silva CM, Castro SV, Luz HK, Rossetto R, et al. Influence of vitrification techniques and solutions on the morphology and survival of preantral follicles after in vitro culture of caprine ovarian tissue. Theriogenology 2011;76:933-41.

[15] Haidari K, Salehnia M, Rezazadeh Valojerdi M. The effect of leukemia inhibitory factor and coculture on the in vitro maturation and ultrastructure of vitrified and nonvitrified isolated mouse preantral follicles. Fertil Steril 2008;90:2389-97.

[16] Lin T-C, Kuo T-C, Lee W-T. EGF and IGF-1 improve maturation of the in-vitro grown cumulus-oocyte-complexes from vitrification preantral follicles. Fertil Steril 2008;90:S292.

[17] Liu J, Van der Elst J, Van den Broecke R, Dhont M. Live offspring by in vitro fertilization of oocytes from cryopreserved primordial mouse follicles after sequential in vivo transplantation and in vitro maturation. Biol Reprod 2001;64:171-8.

[18] Hasegawa A, Mochida N, Ogasawara T, Koyama K. Pup birth from mouse oocytes in preantral follicles derived from vitrified and warmed ovaries followed by in vitro growth, in vitro maturation, and in vitro fertilization. Fertil Steril 2006;86:1182-92.

[19] Jewgenow K, Penfold LM, Meyer HH, Wildt DE. Viability of small preantral ovarian follicles from domestic cats after cryoprotectant exposure and cryopreservation. J Reprod Fertil 1998;112: 39-47.

[20] Jewgenow K. Impact of peptide growth factors on the culture of small preantral follicles of domestic cats. Theriogenology 1996;45: 889-95.

[21] Jewgenow K, Göritz F. The recovery of preantral follicles from ovaries of domestic cats and their characterisation before and after culture. Anim Reprod Sci 1995;39:285-97.

[22] Jewgenow K, Pitra C. Hormone-controlled culture of secondary follicles of domestic cats. Theriogenology 1993;39:527-35.

[23] Wiedemann C, Zahmel J, Jewgenow K. Short-term culture of ovarian cortex pieces to assess the cryopreservation outcome in wild felids for genome conservation. BMC Vet Res 2013;9:37.

[24] Wongbandue G, Jewgenow K, Chatdarong K. Optimization of alginate gel concentration for in vitro growing of cat preantral follicle in three-dimensional culture system. 3rd International Congress on Controversies in Cryopreservation of stem cells, reproductive cells, tissue and organs (Cryo). Berlin, Germany 2013.

[25] Gigli I, Cushman RA, Wahl CM, Fortune JE. Evidence for a role for anti-Mullerian hormone in the suppression of follicle activation in mouse ovaries and bovine ovarian cortex grafted beneath the chick chorioallantoic membrane. Mol Reprod Dev 2005;71:480-8.

[26] Cushman RA, Wahl CM, Fortune JE. Bovine ovarian cortical pieces grafted to chick embryonic membranes: a model for studies on the activation of primordial follicles. Hum Reprod 2002;17:48-54.

[27] Martinez-Madrid B, Donnez J, Van Eyck AS, Veiga-Lopez A, Dolmans MM, Van Langendonckt A. Chick embryo chorioallantoic membrane (CAM) model: a useful tool to study short-term transplantation of cryopreserved human ovarian tissue. Fertil Steril 2009; 91:285-92.

[28] Isachenko $\mathrm{V}$, Orth $\mathrm{I}$, Isachenko $\mathrm{E}$, Mallmann $\mathrm{P}$, Peters $\mathrm{D}$, Schmidt T, et al. Viability of human ovarian tissue confirmed 5 years after freezing with spontaneous ice-formation by autografting and chorio-allantoic membrane culture. Cryobiology 2013;66:233-8.

[29] Zabielska K, Lechowski R, Król M, Pawłowski KM, Motyl T, Dolka I, et al. Derivation of feline vaccine-associated fibrosarcoma cell line and its growth on chick embryo chorioallantoic membrane - a new in vivo model for veterinary oncological studies. Vet Res Commun 2012;36:227-33.

[30] Gougeon A, Chainy GBN. Morphometric studies of small follicles in ovaries of women at different ages. J Reprod Fertil 1987;81:433-42.

[31] Carrijo Jr OA, Marinho AP, Campos AA, Amorim CA, Bao SN, Lucci CM. Morphometry, estimation and ultrastructure of ovarian 
preantral follicle population in queens. Cells Tissues Organs 2010; 191:152-60.

[32] Ribatti D, Nico B, Vacca A, Roncali L, Burri PH, Djonov V. Chorioallantoic membrane capillary bed: a useful target for studying angiogenesis and anti-angiogenesis in vivo. Anat Rec 2001;264:317-24.

[33] Gospodarowicz D. Fibroblast growth factor: involvement in early embryonic development and ovarian function. Semin Reprod Med 1989;7:21-8.

[34] Glister C, Richards SL, Knight PG. Bone morphogenetic proteins (BMP) $-4,-6$, and -7 potently suppress basal and luteinizing hormone-induced androgen production by bovine theca interna cells in primary culture: could ovarian hyperandrogenic dysfunction be caused by a defect in thecal BMP signaling? Endocrinology 2005; 146:1883-92.

[35] Bristol SK, Woodruff TK. Follicle-restricted compartmentalization of transforming growth factor $\beta$ superfamily ligands in the feline ovary. Biol Reprod 2004:70:846-59.

[36] De Pablo F, Pérez-Villamil B, Serna J, González-Guerrero PR, LópezCarranza A, De La Rosa EJ, et al. IGF-I and the IGF-I receptor in development of nonmammalian vertebrates. Mol Reprod Dev 1993; 35:427-33.

[37] Behl R, Kaul R. Insulin like growth factor 1 and regulation of ovarian function in mammals. Indian J Exp Biol 2002;40:25-30.

[38] Tanpradit N, Chatdarong K. Cat ovarian tissue cryopreservation using a passive cooling device. Thai J Vet Med 2011;41:115-6.

[39] Jewgenow K. Role of media, protein and energy supplements on maintenance of morphology and DNA-synthesis of small preantral domestic cat follicles during short-term culture. Theriogenology 1998;49:1567-77.

[40] Sharma GT, Dubey PK, Meur SK. Survival and developmental competence of buffalo preantral follicles using three-dimensional collagen gel culture system. Anim Reprod Sci 2009;114:115-24.

[41] Rajarajan K, Rao BS, Vagdevi R, Tamilmani G, Arunakumari G, Sreenu M, et al. Effect of various growth factors on the in vitro development of goat preantral follicles. Small Ruminant Res 2006; 63:204-12.

[42] Telfer EE, McLaughlin M, Ding C, Thong KJ. A two-step serum-free culture system supports development of human oocytes from primordial follicles in the presence of activin. Hum Reprod 2008;23: $1151-8$.

[43] Göritz F, Jewgenow K, Meyer HHD. Epidermal growth factor and epidermal growth factor receptor in the ovary of the domestic cat (Felis catus). J Reprod Fertil 1996;106:117-24.

[44] Bosch P, Hernandez-Fonseca HJ, Miller DM, Wininger JD, Massey JB, Lamb SV, et al. Development of antral follicles in cryopreserved cat ovarian tissue transplanted to immunodeficient mice. Theriogenology 2004;61:581-94.

[45] Donnez J, Dolmans M-M, Pellicer A, Diaz-Garcia C, Sanchez Serrano M, Schmidt KT, et al. Restoration of ovarian activity and pregnancy after transplantation of cryopreserved ovarian tissue: a review of 60 cases of reimplantation. Fertil Steril 2013;99:1503-13.

[46] Santos RR. Cryopreservation of caprine ovarian tissue: recovery of gonadal function after auto-transplantation. Ridderkerk: Ridderprint; 2007. 